\title{
The violent interstellar medium of the Magellanic Cloud System
}

\author{
You-Hua $\mathrm{Chu}^{1}$ \\ ${ }^{1}$ Astronomy Department, University of Illinois, 1002 W. Green Street, Urbana, IL 61801, USA \\ email: yhchu@illinois.edu
}

\begin{abstract}
The interstellar gas of the Magellanic System is subject to the harassment of tidal interactions on galaxy-wide scales and stellar energy feedback on sub-galactic scales. H i surveys of the Magellanic System have produced spectacular images of the tidally displaced interstellar gas in the Magellanic Bridge and Streams. Multi-wavelength observations of the interstellar gas in the Magellanic Clouds have revealed gas components in physical conditions ranging from cold molecular cloud to hot ionized coronal gas. While stellar energy feedback is responsible for heating and dispersing interstellar gas, it can also compress ambient cloud to form stars. I will use Chandra, XMM-Newton, FUSE, HST, Spitzer, ATCA, and other ground-based observations to illustrate the interplay among massive stars, interstellar medium, and star formation.
\end{abstract}

Keywords. ISM: bubbles, ISM: evolution, ISM: general, galaxies: ISM, Magellanic Clouds

\section{Introduction: ISM in the Magellanic System}

The tidal interactions among the Magellanic Clouds and the Galaxy have wildly disrupted the interstellar medium (ISM) in this system. As show in the H I Parkes All-Sky Survey (HIPASS), H I is distributed not only within the LMC and SMC, but also in the Magellanic Bridge between them and the leading and trailing Streams outside them (Putman et al. 1998). Interstellar absorption observations of abundances in the Magellanic Streams rule out a primordial origin of the gas (Gibson et al. 2000), and suggest that the gas has been pulled out of the SMC (Sembach et al. 2001).

Since the last IAU Symposium on the Magellanic Clouds in 1998, the Australia Telescope Compact Array (ATCA) surveys of H I in the LMC and SMC have been combined with Parkes single-dish observations to recover large-scale diffuse emission. Figure 1 shows the new H I column density maps of the SMC (Stanimirovic et al. 2004) and the LMC (Kim et al. 2003; Staveley-Smith et al. 2003). The effects of tidal interactions are clearly evidenced in the position-velocity plots of the SMC (Fig. 2-left), where double velocity components are seen everywhere. The position-velocity plots of the LMC show high-velocity clouds (HVCs); while some HVCs result from tidal interactions, others are associated with supergiant shells, such as LMC 3 and LMC 8, where stellar energy feedback may be responsible for the HVCs (see Fig. 2-right; Staveley-Smith et al. 2003).

In the bodies of the Magellanic Clouds, over scales of a few to $1000 \mathrm{pc}$, the structure of the ISM is mainly determined by stellar energy feedback via fast stellar winds, supernova explosions, and UV radiation. The energetic interactions have produced multiple phase components of the ISM, which require observations at multiple wavelengths. For example, cold molecular gas requires mm-wavelength $\mathrm{CO}$ observations, interstellar dust requires far-IR observations, H I gas requires $21-\mathrm{cm}$ line observations, warm $\left(10^{4} \mathrm{~K}\right)$ ionized gas is best observed with optical recombination and forbidden lines, hot $\left(10^{6}-10^{7} \mathrm{~K}\right)$ ionized gas requires $\mathrm{X}$-ray observations, and the interface between hot ionized gas and cooler gas is best studied in far-UV. 

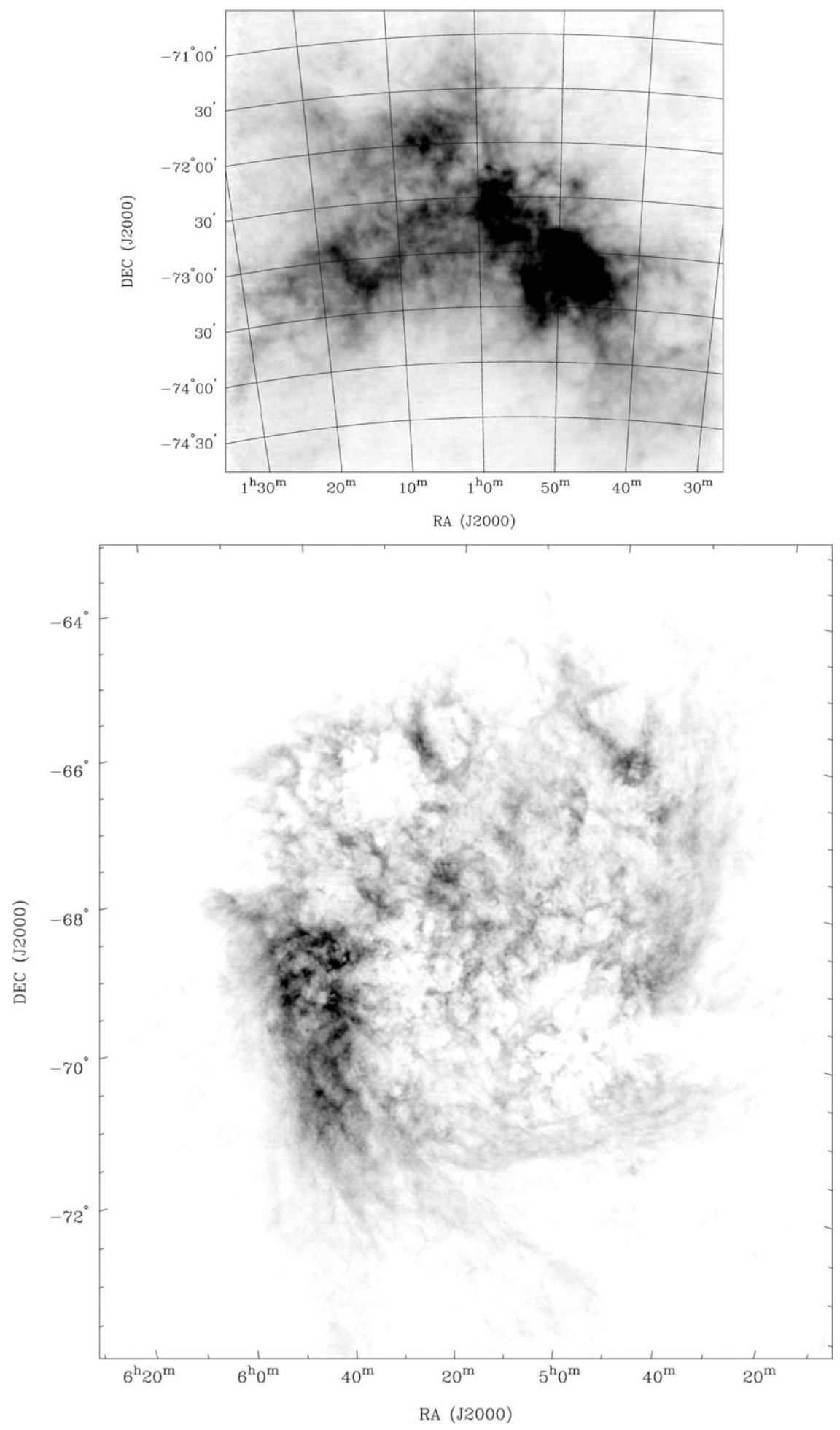

Figure 1. H I column density maps of the SMC (top) and the LMC (bottom) from Stanimirović et al. (2004) and Kim et al. (2003), respectively. 

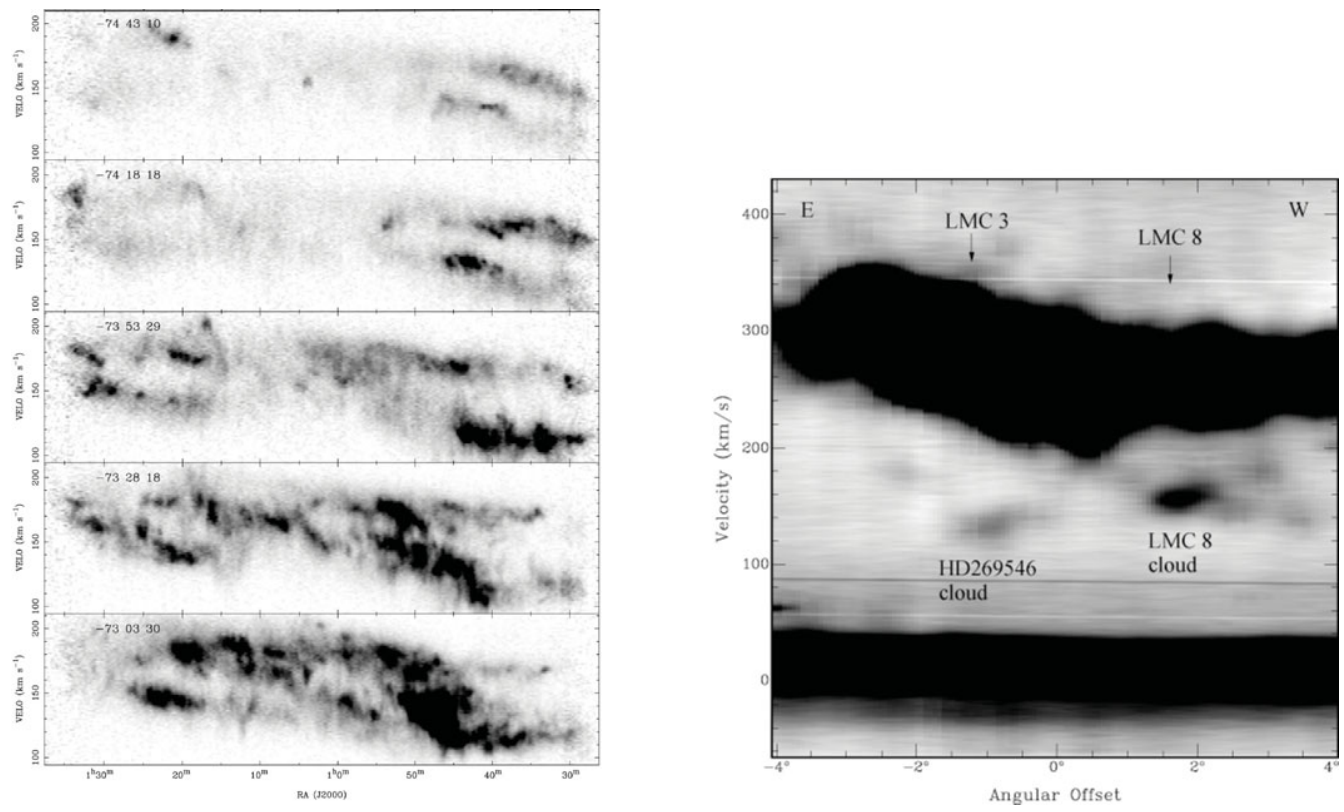

Figure 2. H I position-velocity plots of the SMC (left) from Stanimirović et al. (2004) and the LMC (right) from Staveley-Smith et al. (2003). High-velocity clouds are observed at $\sim 150 \mathrm{~km}$ $\mathrm{s}^{-1}$ toward the supergiant shells LMC 3 and LMC 8.

\section{ISM surveys in the last decade}

The most exciting surveys of the ISM in the Magellanic Clouds are perhaps the Spitzer observations of the dust component in the MIPS bands at 24, 70, and $160 \mu \mathrm{m}$ and IRAC bands at 3.6, 4.5, 5.8, and $8.0 \mu \mathrm{m}$. The Spitzer survey of the LMC, aka SAGE (Meixner et al. 2006), has been used to study dust properties (Bernard et al. 2008) and star formation (Whitney et al. 2008; Gruendl \& Chu 2008). The Spitzer survey of the SMC, aka $\mathrm{S}^{3} \mathrm{MC}$ (Bolatto et al. 2007), has also been used to study the dust mass and properties (Leroy et al. 2007) and star formation (in the giant H II region N 66, Simon et al. 2007).

The molecular clouds in the Magellanic Clouds have been extensively surveyed with the NANTEN telescope (LMC: Fukui et al. 2001; SMC: Mizuno et al. 2001a), and molecular clouds in the LMC have been cataloged by Mizuno et al. (2001b). This first survey has been extended to NANTEN II, which reaches twice the sensitivity of the first survey and is able to detect molecular clouds with masses as low as $\sim 2 \times 10^{4} M_{\odot}$ (Fukui et al. 2008). The NANTEN telescope has also been used to survey the Magellanic Bridge and detected molecular clouds that could be sites for star formation (Mizuno et al. 2006).

The Australian Mopra 22-m telescope and ESO SEST 15-m telescope were used to make the first detection of molecular clouds in the Magellanic Bridge (Muller et al. 2003a). The Mopra telescope is being used to map the molecular clouds in the LMC with higher angular resolution (PI: Tony Wong).

The $A T C A+$ Parkes H I survey of the LMC has been presented and analyzed by Kim et al. (2003) and Staveley-Smith et al. (2003), and the survey of the SMC by Stanimirovic et al. $(1999,2004)$. The survey of H I in the Magellanic Bridge has been used to study its shell population and analyze its power spectrum (Muller et al. 2003b, 2004).

The Magellanic Clouds Emission-Line Survey (MCELS; Smith \& the MCELS Team 1999) of the warm $\left(10^{4} \mathrm{~K}\right)$ ionized gas in the Magellanic Clouds has been completed. The spectacular color composites of the $\mathrm{H} \alpha$, [O III], and [S II] images are available at 
http://www.noao.edu/image_gallery/html/im0993.html (SMC, made by F. Winkler et al.), and http://www.noao.edu/image_gallery/html/im0994.html (LMC, made by C. Smith, S. Points, et al.).

ROSAT surveys of large-scale diffuse X-ray emission from hot gas in the LMC have been reported by Points et al. (2001) and Sasaki et al. (2002). The Chandra X-ray Observatory and the XMM-Newton X-ray Observatory have higher sensitivity and angular resolution, but their small field-of-views make it difficult to carry out large-scale surveys. Observations have been made only for regions of interest, such as 30 Dor (Dennerl et al. 2001; Townsley et al. 2006).

The Far Ultraviolet Spectroscopic Explorer (FUSE) has provided a great opportunity to use interstellar O vi $\lambda \lambda 1031.9,1037.6 \AA$ lines to probe the $10^{5} \mathrm{~K}$ gas at interfaces between $10^{6} \mathrm{~K}$ gas and cooler gas. An atlas of early FUSE spectra of Magellanic Clouds targets was presented by Danforth et al. (2002). The FUSE Magellanic Clouds Legacy Project (http://archive.stsci.edu/prepds/fuse_mc/) has made spectra available for 186 sightlines in the LMC and 100 sightlines in the SMC (Blair et al. 2007). FUSE observations have detected shocked gas in supernova remnants (Blair et al. 2006), interfaces in superbubbles (Sankrit \& Dixon 2007), and hot gas halos (Howk et al. 2002) of the Magellanic Clouds, as well as ionized gas in the Magellanic Bridge (Lehner et al. 2008) and the Magellanic System outside the Clouds themselves (Dixon \& Sankrit 2008).

\section{Stellar energy feedback and star formation}

Massive stars inject energy into the ISM and dynamically change the distribution and physical conditions of the ambient ISM. The interactions frequently produce interstellar shell structures with sizes that depend on the star formation history and distribution of massive stars. Single massive stars can blow bubbles with sizes up to a few $\times 10$ pc, and the bubble ages are observed to be $10^{3}-10^{5}$ yr. When a single massive star explodes as a supernova, the ejecta will interact with the ambient medium and form a supernova remnant (SNR). Multiple massive stars in an OB association can jointly blow a superbubble with diameter up to $\sim 10^{2}$ pc; superbubble ages are commonly observed to be of order $10^{6}$ yr. Supernovae that have exploded in the central cavity of a superbubble will not produce strong optical forbidden line emission or nonthermal radio emission that are characteristic of isolated SNRs; only X-ray emission from the shock-heated, $10^{6}-10^{7} \mathrm{~K}$, gas can be detected. When multiple generations of massive star formation have occurred, a supergiant shell (SGS) with size approaching $10^{3}$ pc can be produced; the dynamic ages of such SGSs are usually a few $\times 10^{7} \mathrm{yr}$.

The interstellar shell structures are prevalent in the LMC, as shown in the MCELS $\mathrm{H} \alpha$ image in Figure 3 (top). A close-up of the SGS LMC 4 region is shown in the bottom panels: the $\mathrm{H} \alpha$ image to the left adequately illustrates the relative sizes of SGS, superbubble, bubble, and SNR, while the H I column density map to the right shows a neutral shell exterior to the ionized SGS LMC 4. The rim of the SGS LMC 4 is dotted with $\mathrm{OB}$ associations and $\mathrm{H}$ II regions with ages of a few Myr, suggesting that the star formation may have been triggered by the expansion of the SGS. Interestingly, the pattern that stellar energy feedback leads to star formation is observed at many different scales.

\subsection{SNRs and star formation}

Star formation has been observed to take place in the vicinity of SNRs. It is unlikely that SNR shocks have triggered the star formation, because the identifying characteristics of SNRs are produced by strong shocks and strong shocks disrupt molecular clouds rather than compressing them to form stars. In H II regions around OB associations, SNRs may 

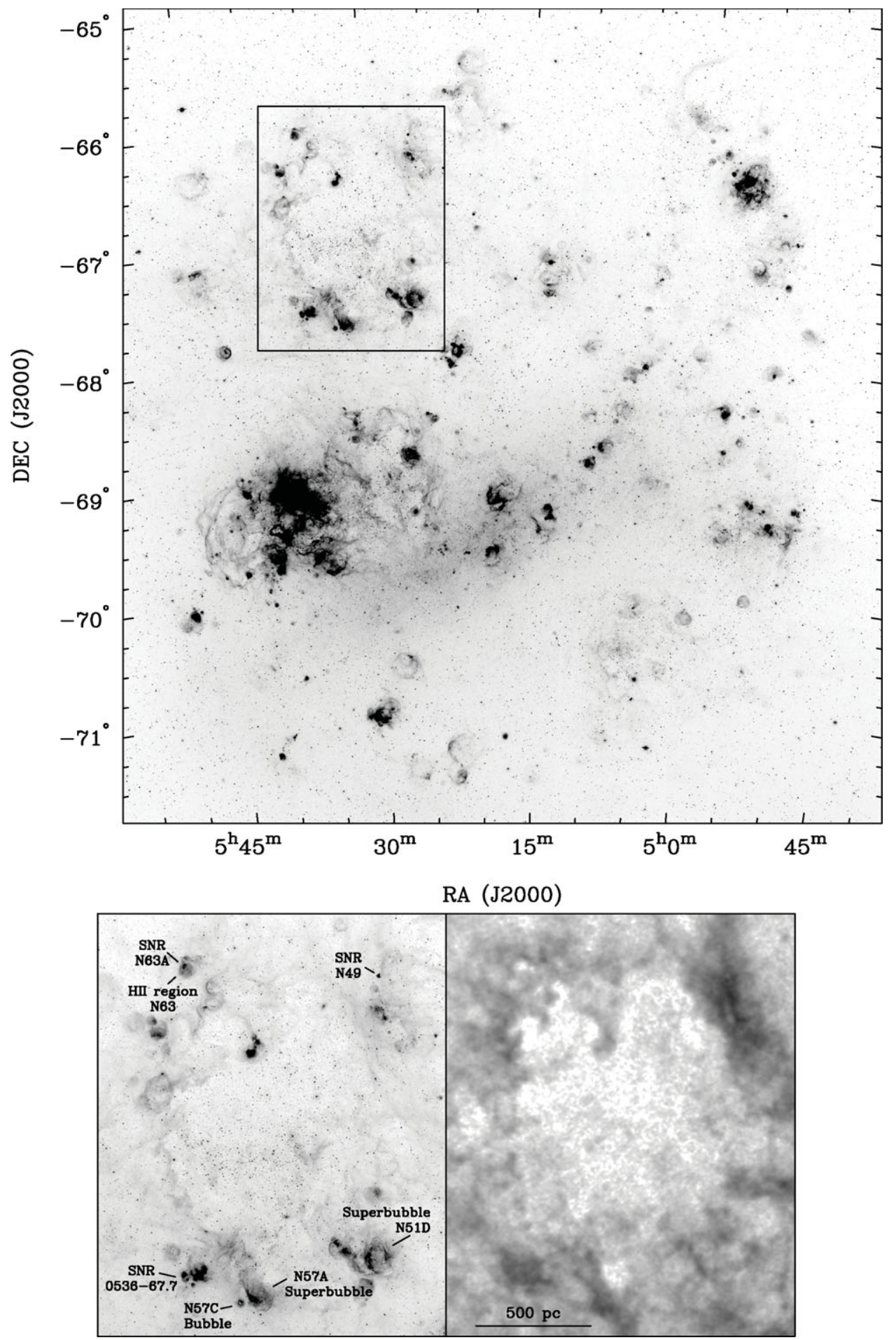

Figure 3. MCELS H $\alpha$ image of the LMC (top). A closeup of the SGS LMC 4 region is shown in the bottom left panel with examples of bubbles and SNRs marked. The H I column density map in the bottom right panel shows a neutral shell exterior to the ionized shell of SGS LMC 4 . 


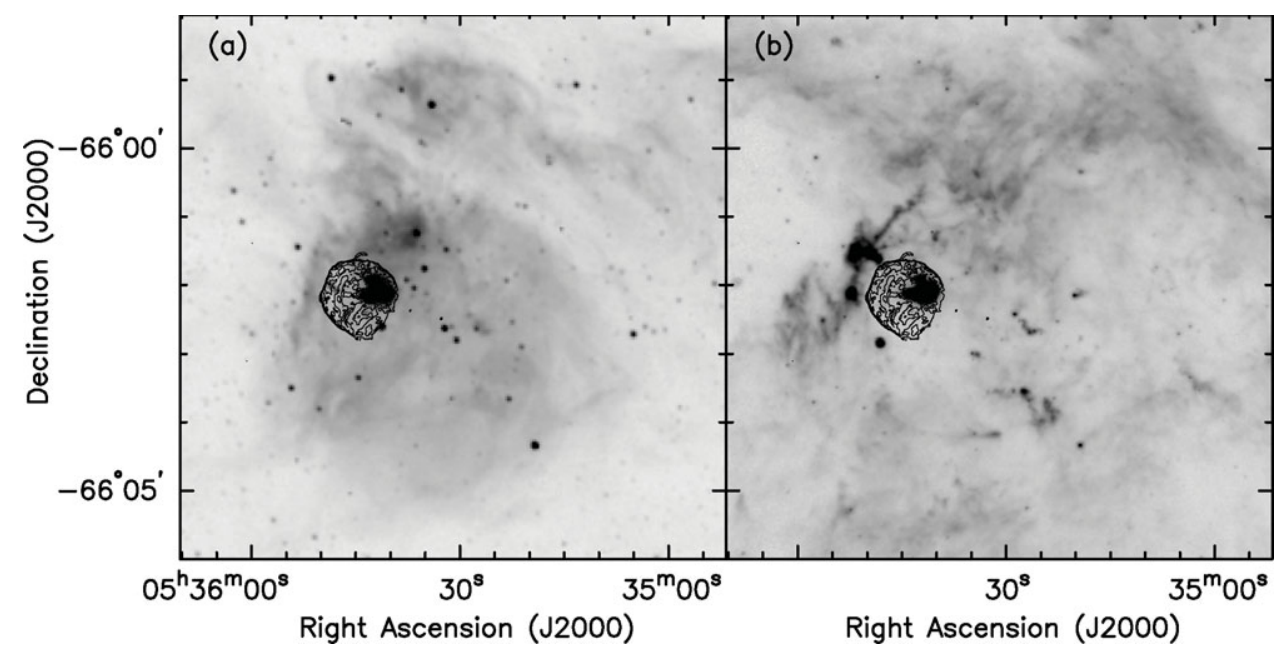

Figure 4. MCELS H $\alpha$ (left) and Spitzer IRAC $8.0 \mu \mathrm{m}$ (right) images of N 63. Overplotted are X-ray contours, extracted from a Chandra ACIS observation, to show the location of the SNR N 63A. Young stellar objects are detected along the northeast boundary of the H II region. The SNR is obviously not responsible for triggering the formation of these young stars.
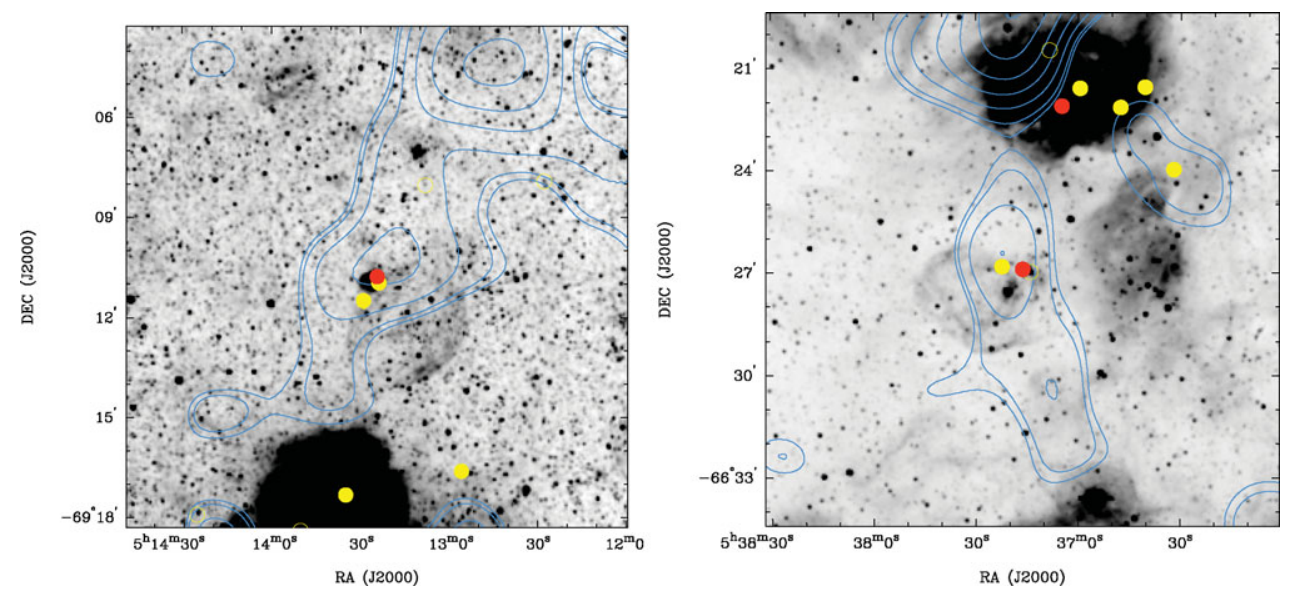

Figure 5. MCELS H $\alpha$ images of SNRs 0513-69.2 (left) and DEML256 (right). Overplotted are NANTEN CO contours. Young stellar objects are marked by filled circles. Young stellar objects are observed along the rim of the SNR projected within a molecular cloud.

exist near young stars and the supernova ejecta may even enrich the star- and planetforming material, but the SNR shocks are not responsible for the onset of star formation. It is more likely that the gentle expansion of an $\mathrm{H}$ II region compresses ambient clouds and triggers star formation. As shown in Figure 4, young stellar objects (YSOs) are present along the rim of the H II region N 63, but not the SNR N 63A (Caulet et al. 2008). The YSOs are just superposed near the SNR N 63A by chance.

Some isolated SNRs show YSOs along their rims, for example, SNRs 0513-69.2 and DEML256 in Figure 5. These two SNRs not only have YSOs, but also appear to be interacting with molecular clouds. The large sizes, $\sim 50 \mathrm{pc}$ in diameter, suggest that they are old $\left(>10^{5} \mathrm{yr}\right)$ SNRs, but their X-ray emission suggests that they are still young $\left(10^{3}-10^{4} \mathrm{yr}\right)$. Only a supernova explosion within the cavity of a pre-existing shell can explain both the large size and X-ray emission. It is most likely that the supernova 
progenitor blew a bubble, the expanding bubble compressed the ambient molecular cloud to form stars, the supernova exploded in the bubble interior, and the supernova ejecta expands quickly through in a low-density medium to reach the dense shell wall. Thus, the supernova progenitors, rather than the SNRs, were responsible for triggering the star formation. Similar phenomenon has been observed in SNRs in the Galaxy (Koo et al. 2008) and near the giant H II region N 66 in the SMC (Gouliermis et al. 2008).

\subsection{Energy feedback and star formation in superbubbles}

The dynamic impact of fast stellar winds and supernova explosions from OB associations can be well illustrated by the multi-wavelength observations of the H II complex N 11 in the LMC (see Figure 6). The $\mathrm{H} \alpha$ image shows a superbubble around the OB association LH 9 at the center. Two bright H II regions, each containing a young OB association, exist along the superbubble shell rim. The ionized gas in the surrounding regions is highly filamentary. A $300 \mathrm{ks}$ Chandra ACIS observation shows diffuse soft X-ray emission not only in the superbubble interior, but also in the surrounding areas. The ATCA+Parkes $\mathrm{H}$ I data show the expansion of the central superbubble and additional expanding structures to the north. Clearly, the ISM in N 11 has been highly disturbed by the energies injected by massive stars. There are still molecular clouds in N 11, and star formation is still raging on: numerous YSOs are observed in regions where the superbubble shell expands into molecular clouds ( $8 \mu \mathrm{m}$ point sources along the superbubble shell rim).

The hot gas content of superbubbles in the LMC has been surveyed with ROSAT observations (Dunne et al. 2001). A detailed analysis of the superbubble N 51D has been carried out by Cooper et al. (2004); they find that the total kinetic and thermal energy retained in the interstellar gas is only about $1 / 3$ of the stellar mechanical energy injected. A remarkable feature of the X-ray emission from N 51D is that its X-ray spectrum requires a nonthermal component to explain the emission in $1-3 \mathrm{keV}$. Nonthermal X-ray emission has also been detected in the LMC superbubbles 30 Dor C (Bamba et al. 2004; Smith \& Wang 2004) and N 11 (Maddox et al. 2008). N 51D does not have detectable molecular clouds around its superbubble shell rim; however, there is still on-going star formation. Some YSOs are projected within the superbubble; high-resolution Hubble Space Telescope images show that these YSOs are formed in dust globules, possibly triggered by photoimplosion (Chu et al. 2005).

\subsection{Energy feedback and star formation in supergiant shells}

The hot gas content of SGSs in the LMC has been studied with ROSAT observations (Points et al. 2001). The physical structure of the SGSLMC 2 has been analyzed in great detail (Points et al. 1999, 2000). Using the improved ATCA+Parkes H I data of the LMC, Book et al. (2008) re-examined the physical structure of all nine optically identified SGSs in the LMC. As shown in Figure 7, the H I structure of LMC 2 is quite complex exhibiting expansion over scales that extend beyond the optical boundaries of the SGS. XMM-Newton observations of LMC 2 detect diffuse X-ray emission from the SGS interior. There is a good correlation between low X-ray surface brightness and high $\mathrm{H}$ I column density, which can be used to determine their relative locations along the line of sight.

Only SGSs LMC 1, 4, 5, and 6 show simple expansion, making it possible to assess their relationship with the on-going star formation (Book et al. 2009). Comparing the YSO and molecular cloud contents of these simple SGSs, it is concluded that triggered star formation is prevalent. Figure 8 shows the SGSs LMC 4 and 5 and their YSOs. There is on-going star formation along the rims of these SGSs and particularly in the interaction zone between these two SGSs. 

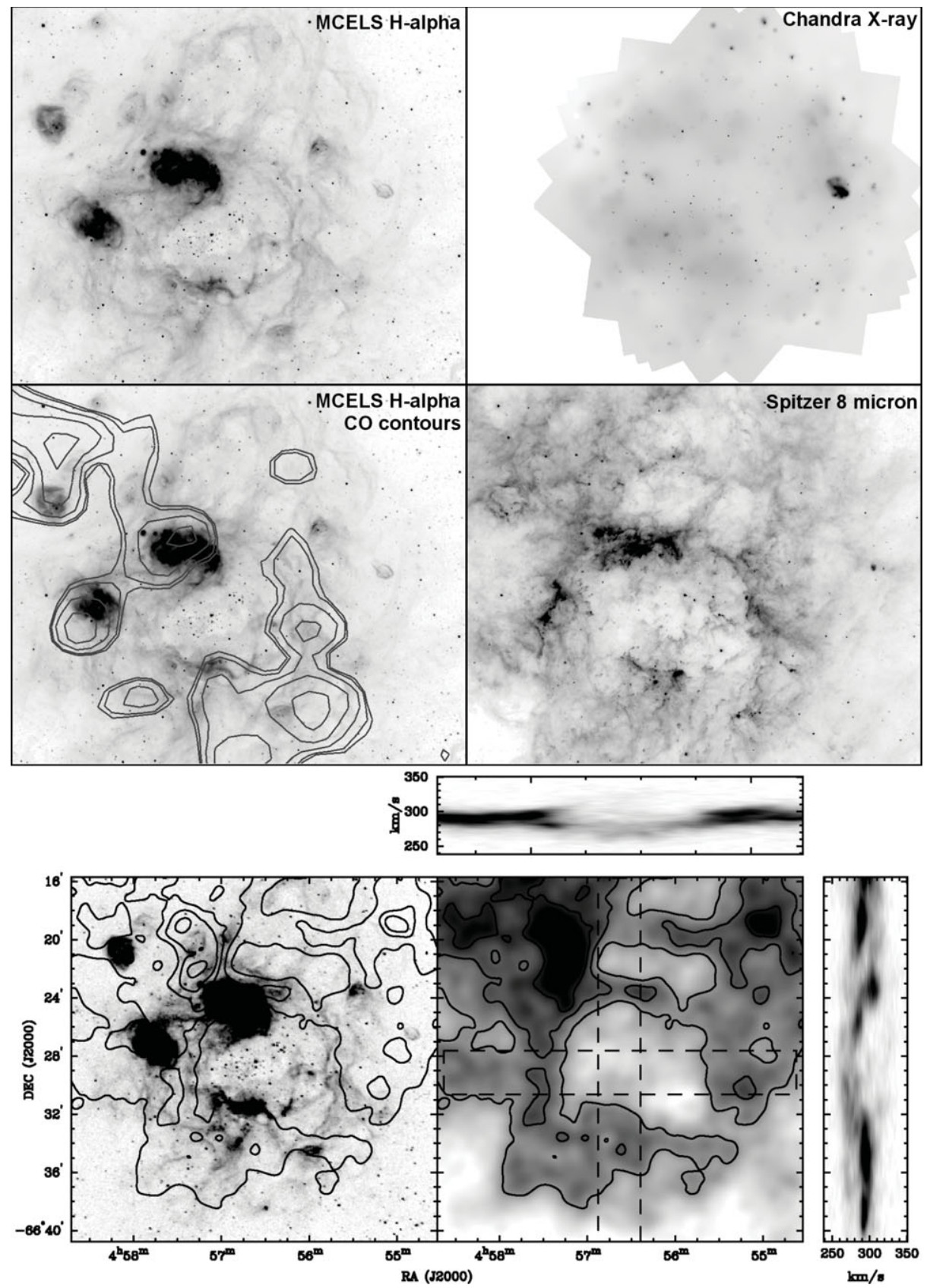

Figure 6. The N 11 H il complex. The top four panels are MCELS H $\alpha$ image, Chandra ACIS image in soft X-rays, $\mathrm{H} \alpha$ image superposed with NANTEN CO contours, and Spitzer IRAC $8.0 \mu \mathrm{m}$ image. The bright X-ray source on the west side of $\mathrm{N} 11$ is the known SNR N 11L. The bottom left panel is an $\mathrm{H} \alpha$ image superposed with $\mathrm{H}$ I contours, and the bottom right panel is an H I column density map extracted from ATCA+Parkes survey (Kim et al. 2003). Two position-velocity plots are presented on the sides of the H I map; the dashed lines in the map mark the locations of the position-velocity plots. 

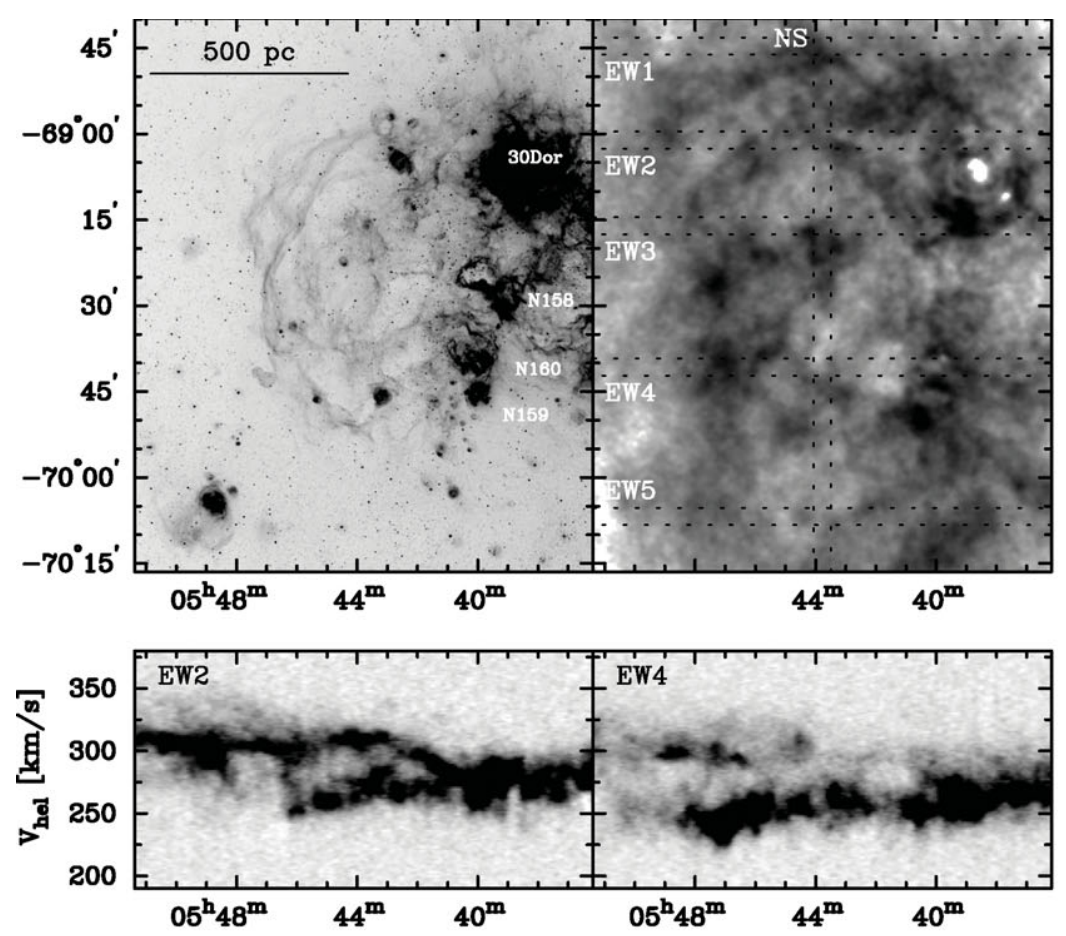

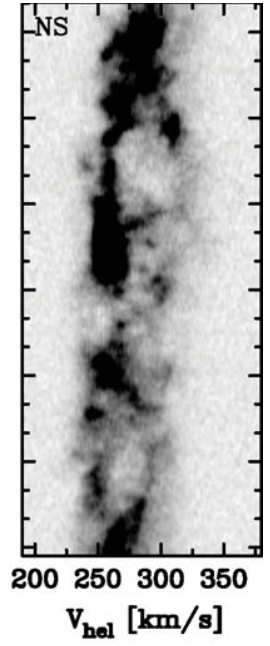

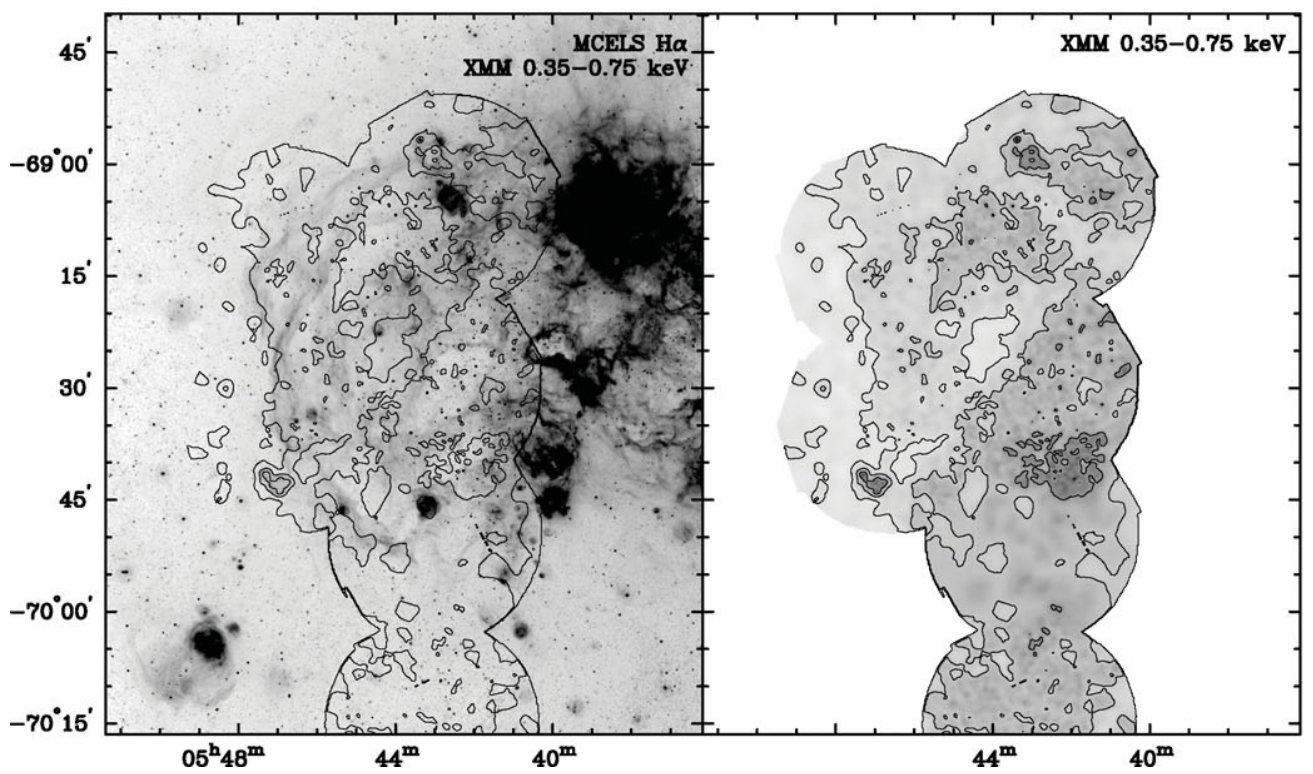

Figure 7. Supergiant shell LMC 2. MCELS H $\alpha$ image and ATCA+Parkes H i column density map. The locations of the position-velocity plots are marked on the $\mathrm{H}$ I map. The lower panels are MCELS $\mathrm{H} \alpha$ image and XMM-Newton X-ray $(0.35-0.75 \mathrm{keV})$ image of LMC 2 ; the X-ray contours are overplotted on both images for comparison. 


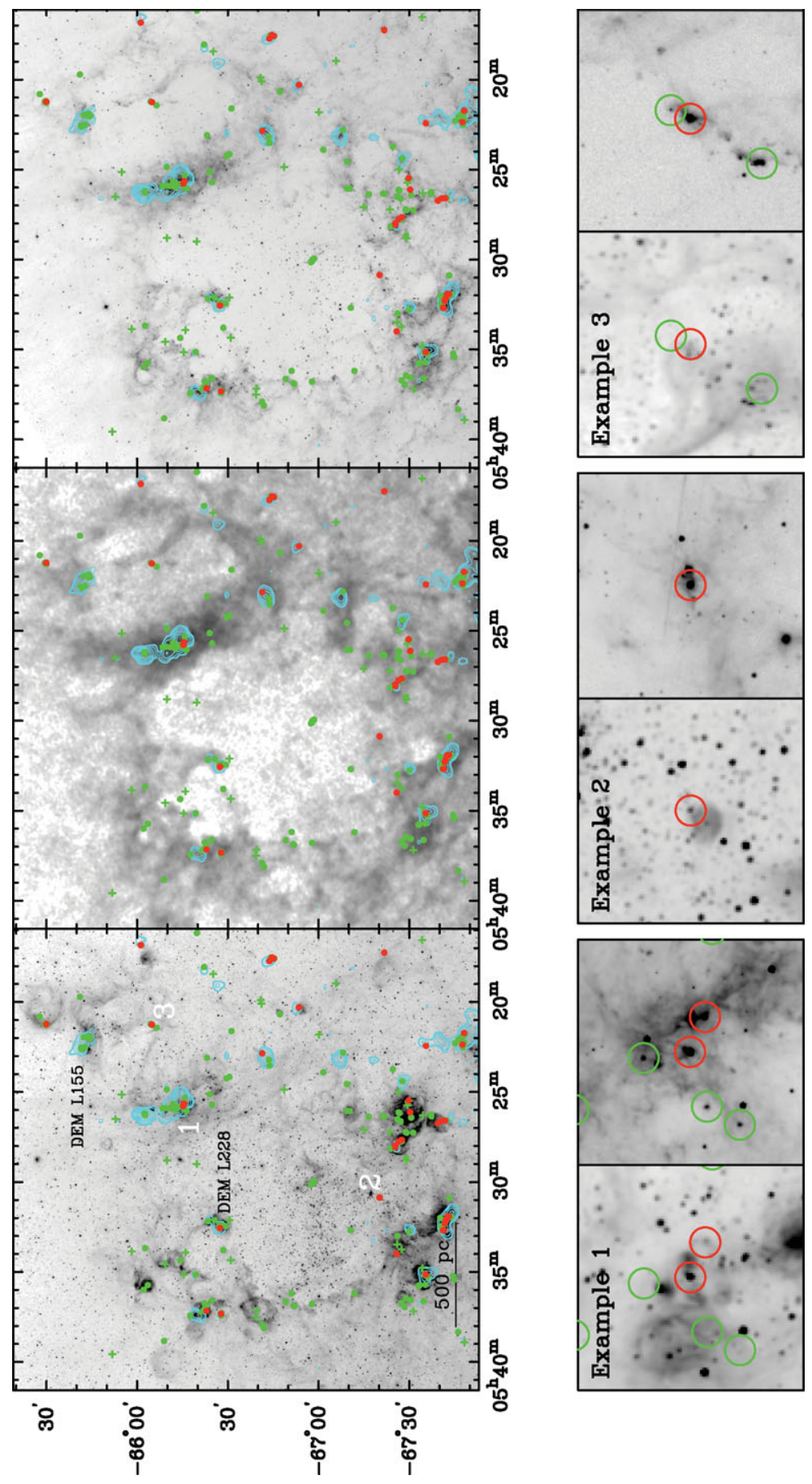

Figure 8. The supergiant shells LMC 4 and 5. Top row (left to right) - MCELS H $\alpha$ image, $A T C A+$ Parkes H I column density map, and Spitzer $8 \mu \mathrm{m}$ image, superposed by NANTEN CO contours and marked with locations of YSOs. The probable YSOs are marked by circles and the less certain ones in crosses. The bottom row shows three examples of YSOs with pairs of $\mathrm{H} \alpha$ (left) and $8 \mu \mathrm{m}$ (right) images. The locations of these examples are marked in the H $\alpha$ image in the top row. This figure is from Book et al. (2009). 


\section{Future work}

The interstellar space is permeated with hot coronal gas, a product of violent shocks from fast stellar winds and supernova explosions. The hot gas component is perhaps the least well studied component of the ISM. While there are X-ray images showing the existence and distribution of hot gas, the spectral information is always of low resolution, which limits our ability to extract accurate temperature and abundance information of the hot gas. Sensitive, high-resolution X-ray spectra of the hot ISM are needed so that the thermal and chemical evolution of the hot gas can be studied with accuracy. It is particularly important to study how supernovae enrich their ambient medium.

\section{Acknowledgements}

This research has been supported by NASA grants SAO GO7-8091A, NNG04GF34G, JPL 1264494, and JPL 1290956. YHC thanks R. Gruendl for making many of the figures in this paper.

\section{References}

Bamba, A., Ueno, M., Nakajima, H., \& Koyama, K. 2004, ApJ, 602, 257

Bernard, J.-P., Reach, W. T., Paradis, D., et al. 2008, AJ, 136, 919

Blair, W. P., Ghavamian, P., Sankrit, R., \& Danforth, C. W. 2006, ApJS, 165, 480

Blair, W. P., et al. 2007, BAAS, 38, 898

Bolatto, A. D., Simon, J. D., Stanimirović, S., et al. 2007, ApJ, 655, 212

Book, L. G., Chu, Y.-H., \& Gruendl, R. A. 2008, ApJS, 175, 165

Book, L. G., Chu, Y.-H., Gruendl, R. A., \& Fukui, Y. 2009, AJ, in press

Caulet, A., Gruendl, R. A., \& Chu, Y.-H. 2008, ApJ, 678, 200

Chu, Y.-H., Gruendl, R. A., Chen, C.-H. R., et al. 2005, ApJ, 634, L189

Cooper, R. L., Guerrero, M. A., Chu, Y.-H., Chen, C.-H. R., \& Dunne, B. C. 2004, ApJ, 605, 751

Danforth, C. W., Howk, J. C., Fullerton, A. W., Blair, W. P., \& Sembach, K. R. 2002, ApJS, 139,81

Dennerl, K., Haberl, F., Aschenbach, B., et al. 2001, A\&A, 365, L202

Dixon, W. V. D. \& Sankrit, R. 2008, ApJ, 686, 1162

Dunne, B. C., Points, S. D., \& Chu, Y.-H. 2001, ApJS, 136, 119

Fukui, Y., Mizuno, N., Yamaguchi, R., Mizuno, A., \& Onishi, T. 2001, PASJ, 53, L41

Fukui, Y., Kawamura, A., Minamidani, T. et al. 2008, PASJ, 178, 56

Gibson, B. K., Giroux, M. L., Penton, S. V., Putman, M. E., Stocke, J. T., \& Shull, J. M. 2000, AJ, 120,1830

Gouliermis, D. A., Chu, Y.-H., Henning, T., Brandner, W., Gruendl, R. A., Hennekemper, E., \& Hormuth, F. 2008, ApJ, 688, 1050

Gruendl, R. A. \& Chu, Y.-Y. 2008, ApJS, submitted

Howk, J. C., Sembach, K. R., Savage, B. D., Massa, D., Friedman, S. D., \& Fullerton, A. W. 2002, ApJ, 569, 214

Kim, S., Staveley-Smith, L., Dopita, M. A., Sault, R. J., Freeman, K. C., Lee, Y., \& Chu, Y.-H. 2003, ApJS, 148, 473

Koo, B.-C., McKee, C. F., Lee, J.-J., et al. 2008, ApJ, 673, L147

Lehner, N., Howk, J. C., Keenan, F. P., \& Smoker, J. V. 2008, ApJ, 678, 219

Leroy, A., Bolatto, A., Stanimirovíc, S., Mizuno, N., Israel, F., \& Bot, C. 2007, ApJ, 658, 1027

Maddox, L. A., Williams, R. M., Dunne, B. C., \& Chu, Y.-H. 2008, ApJ, submitted

Meixner, M., Gordon, K. D., Indebetouw, R., et al. 2006, AJ, 132, 2268

Mizuno, N., Rubio, M., Mizuno, A., Yamaguchi, R., Onishi, T., \& Fukui, Y. 2001a, PASJ, 53, L45

Mizuno, N., Yamaguchi, R., Mizuno, A., et al. 2001b, PASJ, 53, 971 
Mizuno, N., Muller, E., Maeda, H., Kawamura, A., Minamidani, T., Onishi, T., Mizuno, A., \& Fukui, Y. 2006, ApJ, 643, L107

Muller, E., Staveley-Smith, L., \& Zealey, W. J. 2003a, MNRAS, 338, 609

Muller, E., Staveley-Smith, L., Zealey, W., \& Stanimirović, S. 2003b, MNRAS, 339, 105

Muller, E., Stanimirović, S., Rosolowsky, E., \& Staveley-Smith, L. 2004, ApJ, 616, 845

Points, S. D., Chu, Y.-H., Kim, S., Smith, R. C., Snowden, S. L., Brandner, W., \& Gruendl, R. A. $1999, A p J, 518,298$

Points, S. D., Chu, Y.-H., Snowden, S. L., \& Staveley-Smith, L. 2000, ApJ, 545, 827

Points, S. D., Chu, Y.-H., Snowden, S. L., \& Smith, R. C. 2001, ApJS, 136, 99

Putman, M. E., Gibson, B. K., Staveley-Smith, L, et al. 1998, Nature, 394, 752

Sankrit, R. \& Dixon, W. V. D. 2007, PASP, 119, 284

Sasaki, M., Haberl, F., \& Pietsch, W. 2002, A\&A, 392, 103

Sembach, K. R., Howk, J. C., Savage, B. D., \& Shull, J. M. 2001, AJ, 121, 992

Simon, J. D., Bolatto, A. D., Whitney, B. A., et al. 2007, ApJ, 669, 327

Smith, D. A. \& Wang, Q. D. 2004, ApJ, 611, 881

Smith, R. C. The MCELS Team 1999, in New Views of the Magellanic Clouds, 190, 28

Stanimirović, S., Staveley-Smith, L., Dickey, J. M., \& Sault, R. J. 1999, MNRAS, 302, 417

Stanimirović, S., Staveley-Smith, L., \& Jones, P. A. 2004, ApJ, 604, 176

Staveley-Smith, L., Kim, S., Calabretta, M. R., Haynes, R. F., \& Kesteven, M. J. 2003, MNRAS, 339,87

Townsley, L. K., Broos, P. S., Feigelson, E. D., Brandl, B. R., Chu, Y.-H., Garmire, G. P., \& Pavlov, G. G. 2006, AJ, 131, 2140

Whitney, B. A., Sewilo, M., Indebetouw, R., et al. 2008, AJ, 136, 18

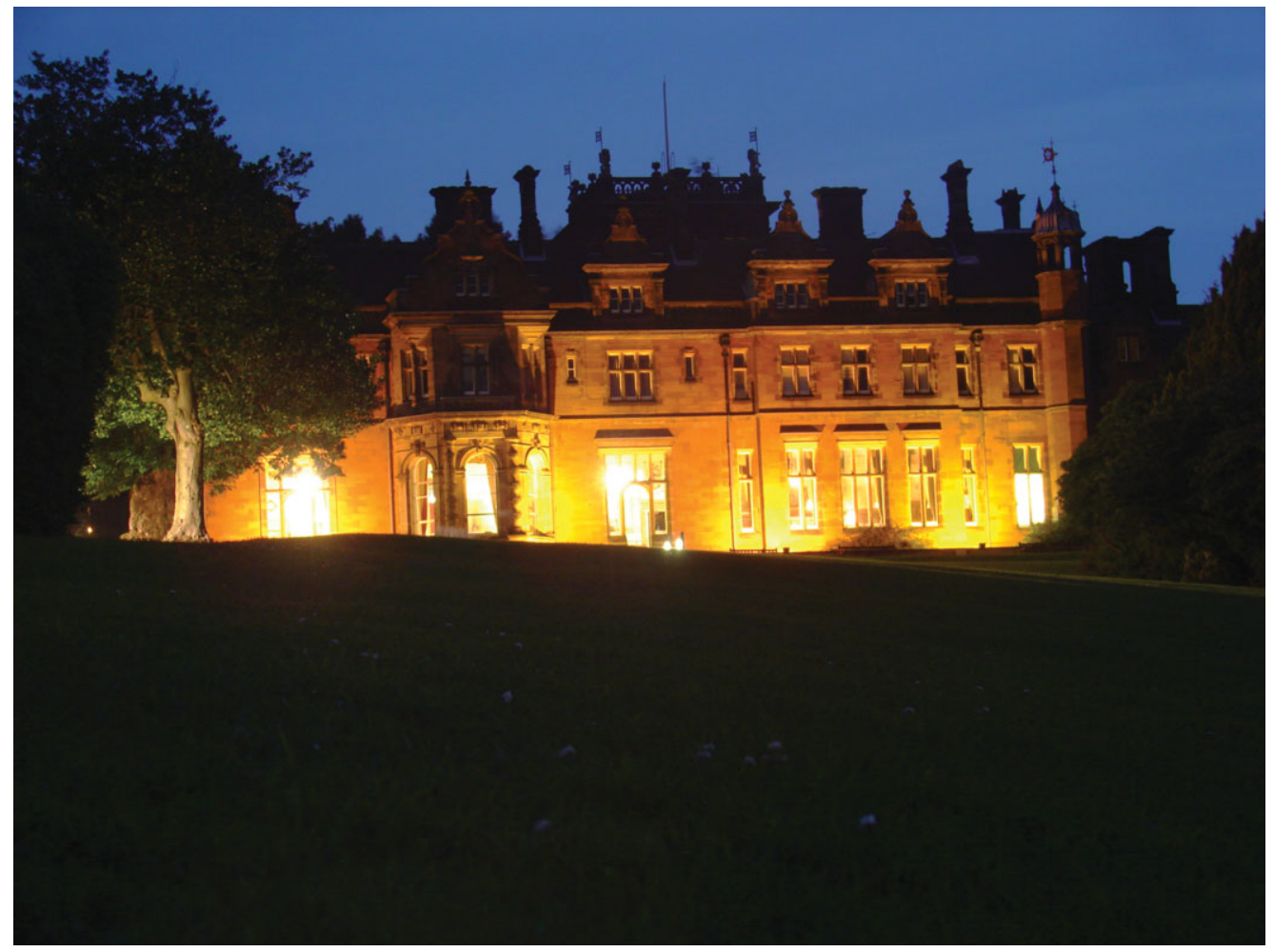

Keele Hall by night. 